\title{
Experimental Parameters of Fine Particle Plasmas Explicitly Expressed by Dimensionless Characteristic Parameters
}

\author{
Hiroo TOTSUJI \\ Graduate School of Natural Science and Technology and Faculty of Engineering, \\ Okayama University, Okayama 700-8530, Japan
}

(Received 15 May 2008 / Accepted 21 June 2008)

\begin{abstract}
Experimental parameters of fine particle plasmas, densities and temperatures of components and the fine particle size, are explicitly expressed in terms of dimensionless characteristic parameters, such as Coulomb coupling and strength of screening, and typical examples are given. Though characteristic parameters are readily obtained from experimental parameters, the reverse is not trivial and it is shown that the charge neutrality condition limits the characteristic parameters realized by fine particle plasmas within the domain $(a / \lambda)^{2} /(\Gamma / A) \geq 3$. Here, $a$ and $\lambda$ are the mean distance between fine particles and the screening length, respectively, $\Gamma=(Q e)^{2} / a k_{\mathrm{B}} T_{\mathrm{p}}$ is the Coulomb coupling of fine particles, and $A=\left(n_{\mathrm{e}} T_{\mathrm{e}}+n_{\mathrm{i}} T_{\mathrm{i}}\right) / n_{\mathrm{p}} T_{\mathrm{p}}$ is the ratio of the ideal gas pressures of ambient plasma (of ions and electrons) and fine particles.
\end{abstract}

(C) 2008 The Japan Society of Plasma Science and Nuclear Fusion Research

Keywords: fine particle (dusty) plasma, dimensionless characteristic parameter, Yukawa one-component plasma (OCP) model, Coulomb coupling

DOI: $10.1585 / \mathrm{pfr} .3 .046$

\section{Introduction}

Fine particle plasmas are mixtures of fine particles (particulates) and ambient plasma of ions and electrons. Fine particle plasmas provide us with a stage for various interesting phenomena especially in the domain of strong coupling such as formation of the Coulomb crystal and other structures. Statistical properties of fine particle plasmas are characterized by a set of several dimensionless characteristic parameters which properly describe the system. In order to observe some phenomenon expected to occur at some specific combination of characteristic parameters, it is necessary to interpret the combination into a set of experimental parameters which realizes the state with designated combination.

When characteristic parameters at a state of fine particle plasmas are obtained in experiment, one may want to modify their values by changing experimental parameters such as the size of fine particles and densities and temperatures of components. Since most of experimental parameters are related to not a single characteristic parameter in the set, it is not trivial to adjust experimental parameters appropriately. Characteristic parameters are readily calculated from experimental parameters by definitions but the reverse needs at least some manipulation and numerical solution of equations. We here give the reverse relations explicitly in a simple form including numerical results to facilitate theoretical and experimental investigations of fine particle plasmas.

In order to make a correspondence between theoretical and experimental parameters, it is necessary to char-

author'se-mail: totsuji@elec.okayama-u.ac.jp acterize statistical-mechanical properties of the system by dimensionless parameters. The simplest model of fine particle plasmas is the Yukawa one-component plasma (Yukawa OCP) model [1-4]. This has a sound basis in the domain of weak coupling and we expect the characterization of the system by its parameters to work at least as a reference frame also in the domain of strong coupling as follows.

There exist some aspects of fine particle plasmas which are not described by the Yukawa OCP model. One is the anisotropy of interaction between particles induced by the effect of gravity on particles. In experiments on the ground, fine particles are kept afloat in the domain of sheath by the electric field. The ion flow in the sheath then leads to anisotropic interactions between particles including (nonreciprocal) attractive part, through the dielectric response [5] or the focusing of ions due to deflections of their orbits by particles $[6,7]$, as observed in the formation of strings of particles along the ion flow. In the directions perpendicular to the flow, on the other hand, the measurements of the force between colliding particles have shown that the interaction is expressed by the Yukawa potential to a good accuracy at least within a few times the screening length [8-10]. The dispersion of oscillations in ordered monolayers of particles can also be fitted to those calculated by the Yukawa interaction [11].

In describing fine particle plasmas, we assume that they can be characterized by parameters for isotropic systems in the first approximation. This may be realized in microgravity experiments [12]. Under this condition, we need not to levitate particles by the sheath electric field which 
causes unidirectional near- or supersonic ion drift. We may also have approximately isotropic system balancing the gravity by, for example, the temperature gradient [13]. Since ions are not drifting in these cases, we expect the screening is mainly provided by ions. After the system is characterized by parameters for isotropic systems and the correspondence to experimental parameters is known, we can take the effects of anisotropy into account.

Apart from the anisotropy, there have also been some arguments that the (isotropic) precipitation of ions onto particles and other mechanisms lead to an additional attractive interaction between them [14]. These effects are expected to appear when some conditions are satisfied and the magnitude of the attraction can become larger than the Yukawa repulsion in the long range domain where the latter is strongly screened. There seem, however, to be no direct experimental observations hitherto. It is also possible to evaluate these effects once the correspondence within the Yukawa OCP characterization is known.

Since the magnitude of the charge on fine particles is typically $10^{3}$ to $10^{4}$ times the elementary charge, the screening around fine particles may not be properly described by the linear Debye-Hückel expression. The screening may also be modified by the collisions of ions forming screening cloud with neutral atoms and other effects, depending on the neutral atom density. In the domain less than a few times the screening length, however, the potential is approximately described by the Yukawa potential [8-10, 15] and the characterization within the Yukawa OCP model may work as a reference frame in considering these effects.

We give a description of fine particle plasmas within the Yukawa OCP model in Sec. 2 and relate characteristic parameters explicitly to experimental parameters in Sec. 3 . Some examples and the discussion on the effect of ionneutral collisions are given in Sec. 4. Concluding remarks are given in Sec. 5 .

\section{Characteristicc Parameters of Fine Particlec Plasmas in Yukawa OCP Modelc}

We consider fine particle plasmas composed of fine particles (particulates) and ambient plasmas of ions and electrons. For simplicity, we assume fine particles are spheres of radius $r_{\mathrm{p}}$ and ions are of single species and with the positive charge $e$. We denote the density, the temperature, and the charge of fine particles by $\left(n_{\mathrm{p}}, T_{\mathrm{p}},-Q e\right)$, assuming that their charge is negative. For ions and electrons, we denote the density and the temperature by $\left(n_{\mathrm{i}}, T_{\mathrm{i}}\right)$ and $\left(n_{\mathrm{e}}, T_{\mathrm{e}}\right)$, respectively. Since it is believed that $T_{\mathrm{e}}>T_{\mathrm{i}}(\sim$ $T_{\mathrm{p}}$ ) in most experiments, we assign different values for the temperatures of the components. We thus have eight parameters for charged components of the system, namely, $\left(r_{\mathrm{p}}, n_{\mathrm{p}}, T_{\mathrm{p}}, Q, n_{\mathrm{i}}, T_{\mathrm{i}}, n_{\mathrm{e}}, T_{\mathrm{e}}\right)$.
We characterize fine particle plasmas by the parameters of the Yukawa OCP model or the one-component Yukawa system embedded in polarizable and neutralizing background plasma of ions and electrons [1-4]. In this model, the potential around a point particle with the charge $-Q e$ is given by the Yukawa potential

$$
\frac{-Q e}{r} \exp (-r / \lambda)
$$

where $\lambda$ is the screening length given by

$$
\frac{1}{\lambda}=\left(\frac{4 \pi n_{\mathrm{i}} e^{2}}{k_{\mathrm{B}} T_{\mathrm{i}}}+\frac{4 \pi n_{\mathrm{e}} e^{2}}{k_{\mathrm{B}} T_{\mathrm{e}}}\right)^{1 / 2} .
$$

In experiments on Earth, the ion flow gives the anisotropy of interaction between fine particles which is neglected in the above model. There exist, however, many aspects of fine particle plasmas within the isotropic interaction and we assume that our system can be regarded as isotropic at least in the first approximation. In recent experiments under microgravity, we may expect much reduced ion flow and anisotropy.

In the Yukawa OCP model, the statistical properties of fine particles are characterized by the strength of the Coulomb coupling

$$
\Gamma=\frac{(Q e)^{2}}{a k_{\mathrm{B}} T_{\mathrm{p}}}=\left(\frac{4 \pi n_{\mathrm{p}}}{3}\right)^{1 / 3} \frac{(Q e)^{2}}{k_{\mathrm{B}} T_{\mathrm{p}}}
$$

and the strength of the screening

$$
\xi=\frac{a}{\lambda} \text {. }
$$

Here $a$ is the mean distance between fine particles defined by

$$
a=\left(\frac{3}{4 \pi n_{\mathrm{p}}}\right)^{1 / 3} .
$$

When the particle have a spherical core of radius $r_{\mathrm{p}}$ and the charge $-Q e$ is uniformly distributed on the surface, the potential at $r>r_{\mathrm{p}}$ is modified into the form $[16,17]$

$$
\begin{aligned}
& \frac{(-Q e)}{1+r_{\mathrm{p}} / \lambda} \frac{\exp \left[-\left(r-r_{\mathrm{p}}\right) / \lambda\right]}{r} \\
& =\frac{(-\tilde{Q} e)}{r} \exp (-r / \lambda) \text { for } r>r_{\mathrm{p}},
\end{aligned}
$$

where

$$
\tilde{Q}=Q \frac{\exp \left(\tilde{r_{\mathrm{p}}}\right)}{1+\tilde{r_{\mathrm{p}}}}
$$

and

$$
\tilde{r_{\mathrm{p}}}=\frac{r_{\mathrm{p}}}{\lambda}
$$

This potential field is equivalent to that of a point particle with the renormalized charge $-\tilde{Q} e$. The interaction between fine particles is given by the Deryagin-LandauVerwey-Overbeek (DLVO) potential which is still of the Yukawa type

$$
\frac{(-\tilde{Q} e)^{2}}{r} \exp (-r / \lambda) \text { for } r>2 r_{\mathrm{p}},
$$

$r$ being the distance between centers $[16,17]$. 


\section{Experimental Parameters in Terms of Characteristic Parametersc}

We now consider the correspondence between the characteristic parameters of fine particle plasmas and experimental parameters of densities and temperatures within the characterization by the Yukawa OCP model parameters. As the latter, we have eight parameters and we need eight conditions to determine them.

\subsection{Conditions}

We first assume that the charge neutrality condition is satisfied:

$$
n_{\mathrm{i}}=n_{\mathrm{e}}+Q n_{\mathrm{p}}
$$

When characteristic parameters $(\Gamma, \xi)$ are specified, we have two conditions:

$$
\left(\frac{4 \pi n_{\mathrm{p}}}{3}\right)^{1 / 3} \frac{(Q e)^{2}}{k_{\mathrm{B}} T_{\mathrm{p}}}=\Gamma
$$

and

$$
\left(\frac{3}{4 \pi n_{\mathrm{p}}}\right)^{1 / 3}\left(4 \pi e^{2}\right)^{1 / 2}\left(\frac{n_{\mathrm{i}}}{k_{\mathrm{B}} T_{\mathrm{i}}}+\frac{n_{\mathrm{e}}}{k_{\mathrm{B}} T_{\mathrm{e}}}\right)^{1 / 2}=\xi .
$$

The charge on a fine particle $-Q e$ is determined by the balance between the fluxes of ions and electrons onto the surface. When we write $Q$ in the form

$$
Q=f_{Q} \frac{k_{\mathrm{B}} T_{\mathrm{e}}}{e^{2} / r_{\mathrm{p}}}
$$

introducing $f_{Q}$, the condition is given by

$$
\begin{aligned}
& n_{\mathrm{e}}\left(\frac{k_{\mathrm{B}} T_{\mathrm{e}}}{m_{\mathrm{e}}}\right)^{1 / 2} \exp \left(-\frac{f_{Q}}{1+\tilde{r}_{\mathrm{p}}}\right) \\
&-n_{\mathrm{i}}\left(\frac{k_{\mathrm{B}} T_{\mathrm{i}}}{m_{\mathrm{i}}}\right)^{1 / 2}\left(1+\frac{f_{Q}}{1+\tilde{r}_{\mathrm{p}}} \frac{T_{\mathrm{e}}}{T_{\mathrm{i}}}\right)=0
\end{aligned}
$$

in the orbital-motion-limited (OML) theory [18]. Here the effect of reduction of the electron density [19] is reflected through (10). We take into account that the surface potential of the fine particles is given by

$$
\begin{aligned}
& {\left[\frac{(-\tilde{Q} e)}{r} \exp (-r / \lambda)\right]_{r=r_{\mathrm{p}}}=\frac{(-\tilde{Q} e)}{r_{\mathrm{p}}} \exp \left(-r_{\mathrm{p}} / \lambda\right)} \\
& =\frac{(-Q e)}{r_{\mathrm{p}}\left(1+\tilde{r_{\mathrm{p}}}\right)} .
\end{aligned}
$$

We discuss the applicability of the OML theory when examples of experimental parameters are given.

Since we have imposed four conditions, (10), (11), (12), and (14) for eight experimental parameters, we are left with four degrees of freedom. As these four, we take the radius $r_{\mathrm{p}}$,

$$
\Gamma_{0}=\frac{(Q e)^{2}}{r_{\mathrm{p}} k_{\mathrm{B}} T_{\mathrm{p}}},
$$

$$
A=\frac{n_{\mathrm{e}} T_{\mathrm{e}}+n_{\mathrm{i}} T_{\mathrm{i}}}{n_{\mathrm{p}} T_{\mathrm{p}}},
$$

and the ratio of the ion and fine particle temperatures

$$
\tau_{\text {ip }}=\frac{T_{\mathrm{i}}}{T_{\mathrm{p}}} .
$$

The value of $A$ is the ratio of the ideal gas pressure of ambient plasma (ions and electrons) to that of fine particles. It is to be noted that

$$
\eta=\left(\frac{\Gamma}{\Gamma_{0}}\right)^{3}=\left(\frac{r_{\mathrm{p}}}{a}\right)^{3}=\frac{4 \pi}{3} n_{\mathrm{p}} r_{\mathrm{p}}^{3}
$$

is the packing fraction of fine particles, which we denote by $\eta$, and

$$
\tilde{r_{\mathrm{p}}}=\frac{\Gamma}{\Gamma_{0}} \xi
$$

In principle, the ratio $\tau_{\text {ip }}$ is determined by other parameters through the energy relaxation processes which include neutral atoms. In most experiments, however, $\tau_{\text {ip }} \sim 1$ is implicitly assumed without detailed analysis of the latter processes or temperature measurements. In this paper we treat this ratio as an externally determined parameter expecting to be around unity, instead of giving other conditions to determine $\tau_{\text {ip }}$.

\subsection{Solutions}

We first note that, from (10), (11), (12), (14), (19), (17), and (18), $n_{\mathrm{p}}, n_{\mathrm{i}} /\left(A / \tau_{\mathrm{ip}}\right), n_{\mathrm{e}} /\left(A / \tau_{\mathrm{ip}}\right), T_{\mathrm{i}} /\left(A / \tau_{\mathrm{ip}}\right)=$ $\tau_{\text {ip }} T_{\mathrm{p}} /\left(A / \tau_{\text {ip }}\right)$, and $T_{\mathrm{e}} /\left(A / \tau_{\text {ip }}\right)$ are explicitly expressed in terms of $r_{\mathrm{p}}, \Gamma / A, \xi, \Gamma / \Gamma_{0}$, and $f_{Q}$ (see Appendix) as

$$
\begin{gathered}
n_{\mathrm{p}}=\frac{3}{4 \pi r_{\mathrm{p}}^{3}}\left(\frac{\Gamma}{\Gamma_{0}}\right)^{3}, \\
\frac{n_{\mathrm{i}} /\left(A / \tau_{\mathrm{ip}}\right)}{n_{\mathrm{p}}}=\frac{f_{Q}^{2}}{\Gamma_{0} / A} \\
\times \frac{\left[1+\left(\Gamma_{0} / A\right) / f_{Q}\right]^{2}}{\left\{\left[f_{Q}^{2} /\left(\Gamma_{0} / A\right)\right]\left[1+\left(\Gamma_{0} / A\right) / f_{Q}\right]+(1 / 3) \xi^{2}\left(\Gamma_{0} / \Gamma\right)+f_{Q}\right\}}, \\
\times \frac{n_{\mathrm{e}} /\left(A / \tau_{\mathrm{ip}}\right)}{n_{\mathrm{p}}}=f_{Q}^{2} \frac{\left[1+\left(\Gamma_{0} / A\right) / f_{Q}\right]}{\left[\left[f_{Q}^{2} /\left(\Gamma_{0} / A\right)\right]\left[1+\xi^{2}\left(\Gamma_{0} / \Gamma\right)+f_{Q}\right]\right.} \\
\frac{\left[(1 / 3) \xi^{2} /(\Gamma / A)-1\right]}{\left(A / \tau_{\mathrm{ip}}\right)}=\tau_{\mathrm{ip}} \frac{\left.\left.\left.T_{\mathrm{p}} / A\right) / f_{Q}\right]+(1 / 3) \xi^{2}\left(\Gamma_{0} / \Gamma\right)+f_{Q}\right\}}{\left(A / \tau_{\mathrm{ip}}\right)} \\
=\frac{f_{Q}^{2}}{\Gamma_{0} / A}\left[\frac{1+\left(\Gamma_{0} / A\right) / f_{Q}}{(1 / 3) \xi^{2}\left(\Gamma_{0} / \Gamma\right)+f_{Q}}\right]^{2} \frac{e^{2}}{r_{\mathrm{p}}},
\end{gathered}
$$

and

$$
\frac{T_{\mathrm{e}}}{\left(A / \tau_{\mathrm{ip}}\right)}=\left[\frac{1+\left(\Gamma_{0} / A\right) / f_{Q}}{(1 / 3) \xi^{2}\left(\Gamma_{0} / \Gamma\right)+f_{Q}}\right] \frac{e^{2}}{r_{\mathrm{p}}} .
$$



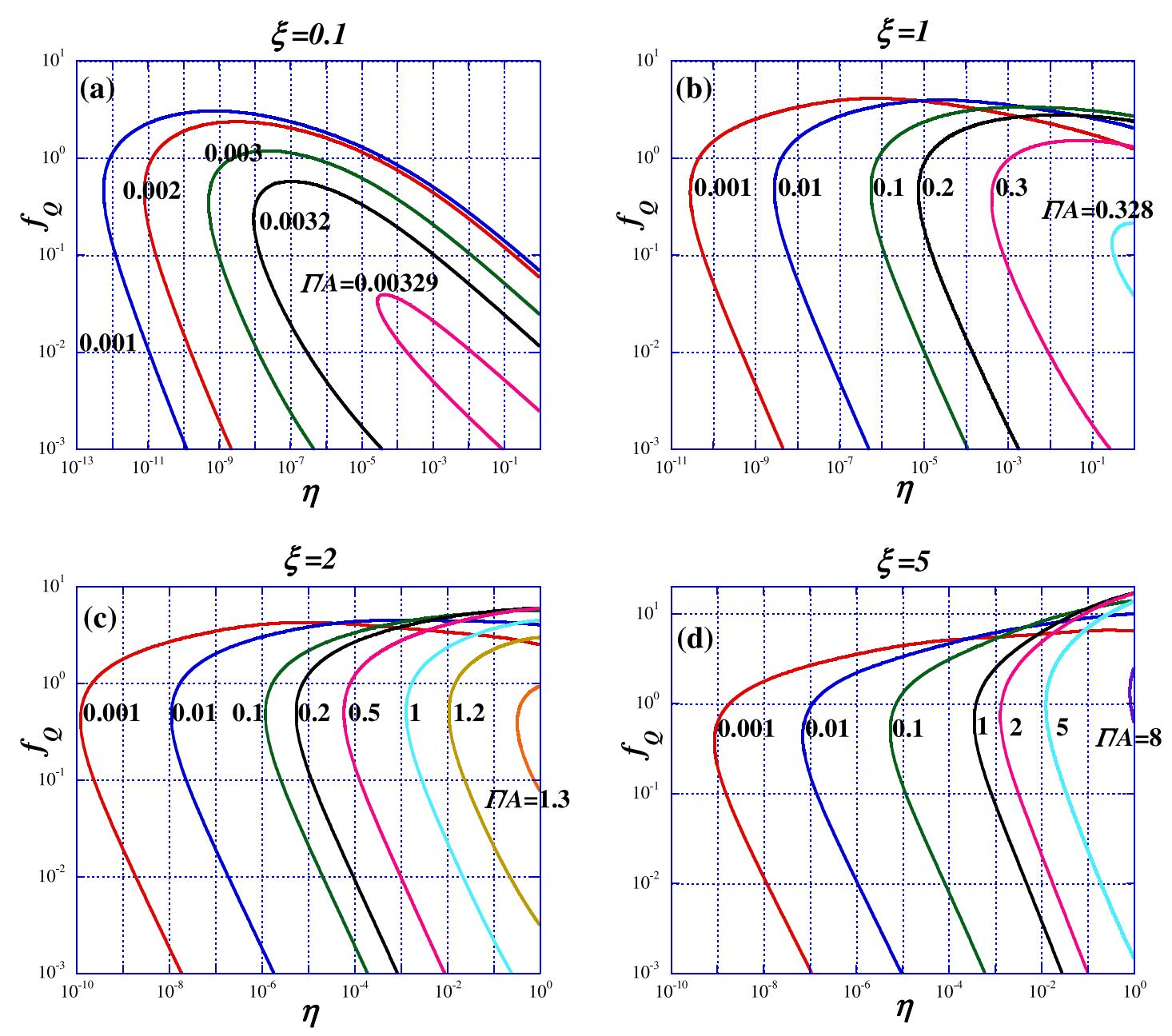

Fig. 1 Values of $f_{Q}$ as function of packing fraction $\eta=\left(\Gamma / \Gamma_{0}\right)^{3}$ for some values of $\xi$ and $\Gamma / A$. (a) $\xi=0.1$ and $0.001 \leq \Gamma / A \leq 0.00329$, (b) $\xi=1$ and $0.001 \leq \Gamma / A \leq 0.328$, (c) $\xi=2$ and $0.001 \leq \Gamma / A \leq 1.3$, and (d) $\xi=5$ and $0.001 \leq \Gamma / A \leq 8$.

We here point out that, since $n_{\mathrm{e}} \geq 0$, we have to satisfy the condition $(1 / 3) \xi^{2} /(\Gamma / A) \geq 1$ or

$$
\xi^{2}=\left(\frac{a}{\lambda}\right)^{2} \geq 3\left(\frac{\Gamma}{A}\right)
$$

This comes from the condition of charge neutrality [19] but has not been explicitly shown in terms of characteristic parameters. Thus the lower limit of realizable $\xi$ is determined by $\Gamma$. Since the value of $A$ is usually many orders of magnitude larger than unity, this lower bound for $\xi$ is not effective in most cases even in the domain of strong coupling where $A \gg \Gamma \gg 1$. In the case of very strong coupling where $\Gamma / A \sim 1$ [20-22], however, this bound does limit the space of characteristic parameters of Yukawa OCP realized by fine particle plasmas.

Substituting (22), (23), (24), (25), and (20) into the equation for $f_{Q}$ or (14), we can determine the value of $f_{Q}$. We note that, since (14) includes only the ratios $n_{\mathrm{e}} / n_{\mathrm{i}}$ and $T_{\mathrm{e}} / T_{\mathrm{i}}$ which are independent of $r_{\mathrm{p}}$ or $A / \tau_{\mathrm{ip}}$, the value of $f_{Q}$ is determined self-consistently when the set of values $\left(\Gamma / A, \xi, \Gamma / \Gamma_{0}\right)$ is specified. Let us now introduce $n_{0}$ and $E_{0}=k_{\mathrm{B}} T_{0}$ defined respectively by

$$
n_{0} \equiv \frac{3}{4 \pi r_{\mathrm{p}}^{3}}
$$

and

$$
E_{0}=k_{\mathrm{B}} T_{0} \equiv \frac{e^{2}}{r_{\mathrm{p}}}
$$

We also define $A^{\prime}$ by

$$
A^{\prime}=\frac{A}{\tau_{\mathrm{ip}}} .
$$

Then the values of $\left(f_{Q}, n_{\mathrm{p}} / n_{0},\left(n_{\mathrm{i}} / n_{0}\right) / A^{\prime},\left(n_{\mathrm{e}} / n_{0}\right) / A^{\prime}\right.$, $\left.\left(T_{\mathrm{i}} / T_{0}\right) / A^{\prime}=\tau_{\text {ip }}\left(T_{\mathrm{p}} / T_{0}\right) / A^{\prime},\left(T_{\mathrm{e}} / T_{0}\right) A^{\prime}\right)$ are determined by the set of values $\left(\Gamma / A, \xi, \Gamma / \Gamma_{0}\right)$ irrespective of the values of $\left(r_{\mathrm{p}}, A / \tau_{\mathrm{ip}}\right)$ :

$$
\begin{aligned}
& \left(\frac{\Gamma}{A}, \xi, \frac{\Gamma}{\Gamma_{0}}\right) \text { determines } \\
& \left(f_{Q}, \frac{n_{\mathrm{p}}}{n_{0}}, \frac{n_{\mathrm{i}}}{A^{\prime} n_{0}}, \frac{n_{\mathrm{e}}}{A^{\prime} n_{0}}, \frac{T_{\mathrm{i}}}{A^{\prime} T_{0}}=\tau_{\mathrm{ip}} \frac{T_{\mathrm{p}}}{A^{\prime} T_{0}}, \frac{T_{\mathrm{e}}}{A^{\prime} T_{0}}\right) \\
& \text { irrespective of }\left(r_{\mathrm{p}}, A / \tau_{\mathrm{ip}}\right) .
\end{aligned}
$$

We may thus regard $r_{\mathrm{p}}, A$, and $\tau_{\text {ip }}$ as a kind of adjustable parameters which can be chosen so as to satisfy the conditions for densities or temperatures. 


\section{$\Pi A=0.003, \xi=0.1$}

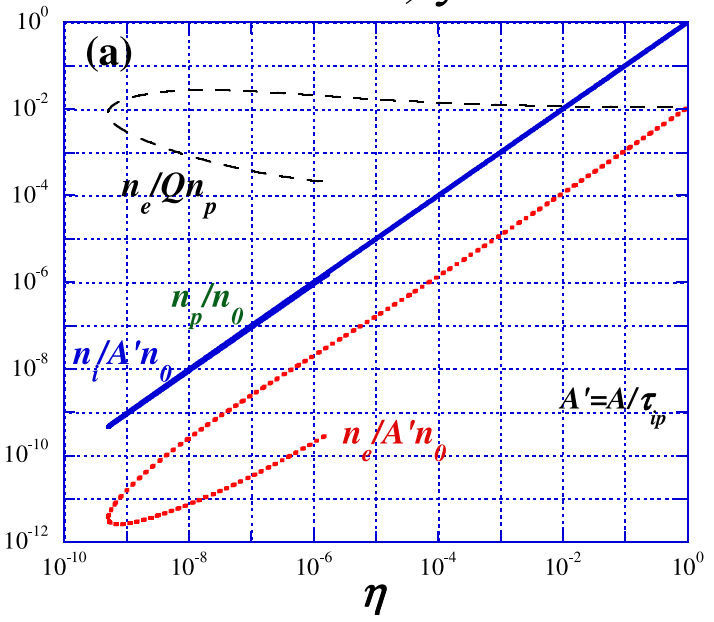

$\Pi / A=0.1, \xi=1$

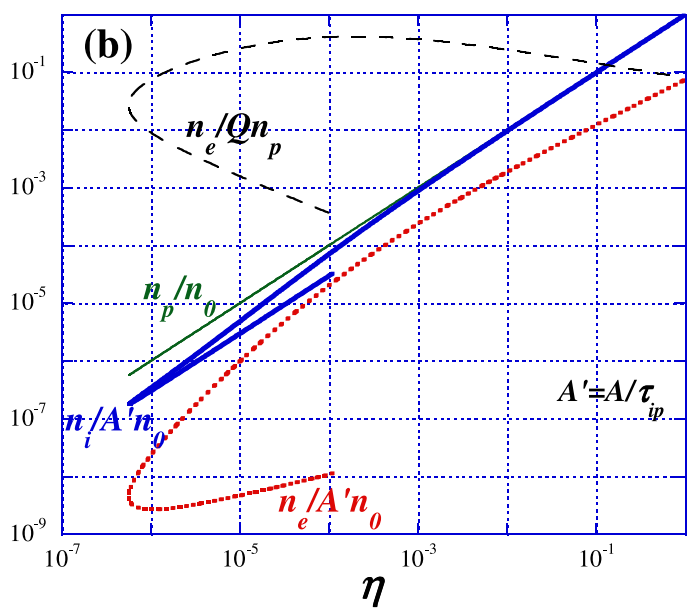

$\Pi A=0.1, \xi=5$

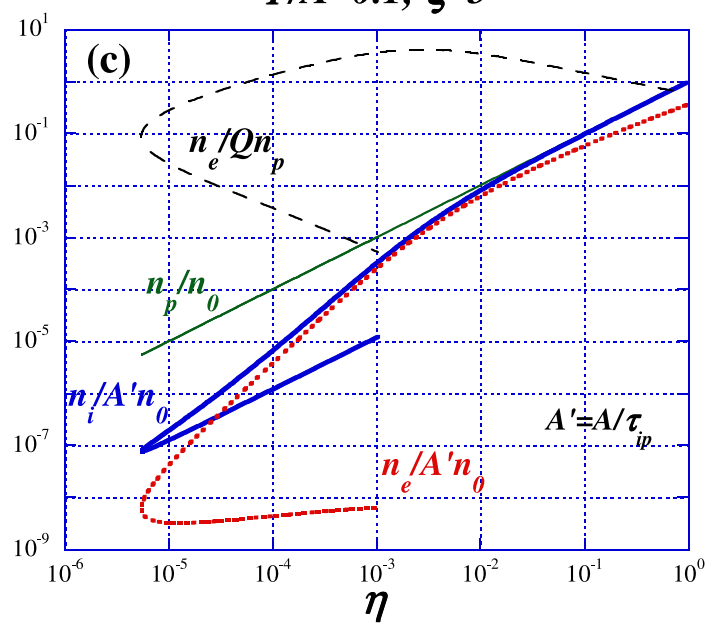

$\Pi A=0.003, \xi=0.1$

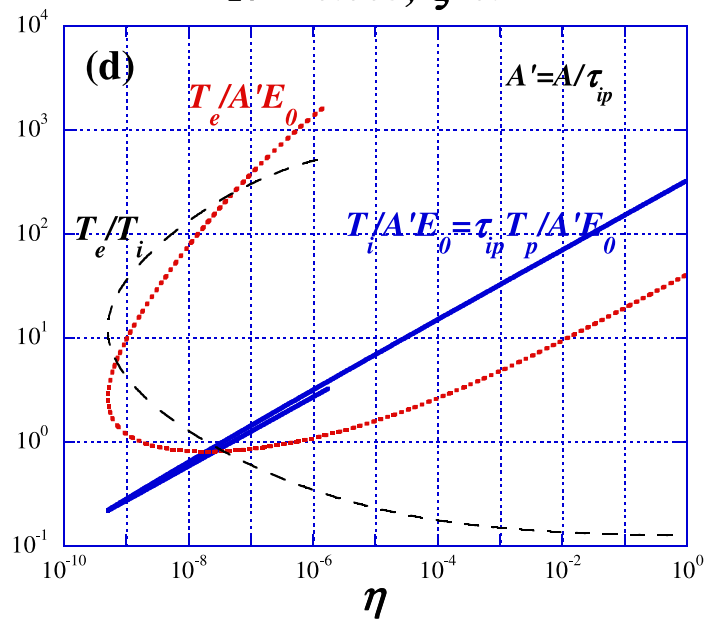

$\Pi A=0.1, \xi=1$

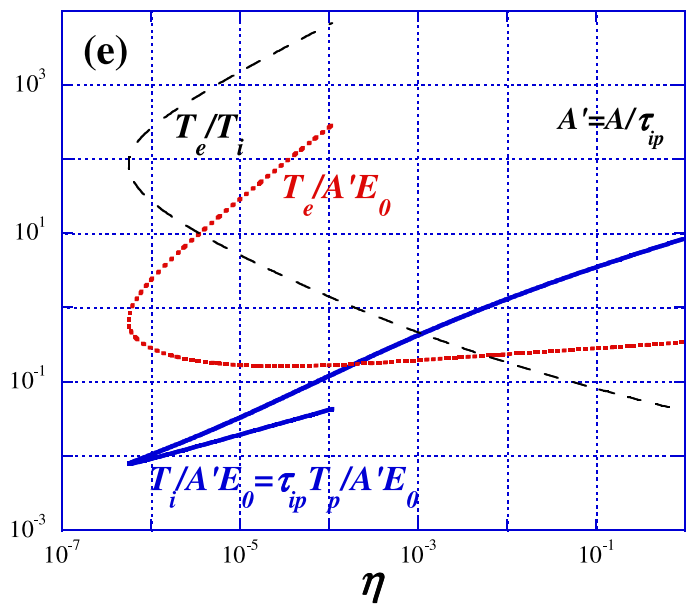

$\Pi A=0.1, \xi=5$

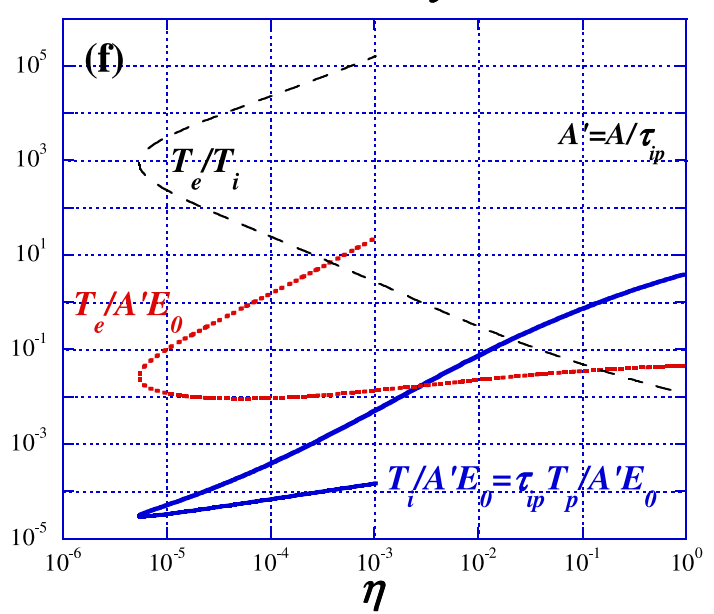

Fig. 2 (a-c) Values of $\left(n_{\mathrm{i}} / n_{0}\right) / A^{\prime}$ (thick solid line), $\left(n_{\mathrm{e}} / n_{0}\right) / A^{\prime}$ (dotted line), $n_{\mathrm{p}} / n_{0}$ (thin solid line) and $n_{\mathrm{e}} / Q n_{\mathrm{p}}$ (broken line). (d-f) Values of $\left(T_{\mathrm{i}} / T_{0}\right) / A^{\prime}\left(=\tau_{\mathrm{ip}}\left(T_{\mathrm{p}} / T_{0}\right) / A^{\prime}\right.$, solid line), $\left(T_{\mathrm{e}} / T_{0}\right) / A^{\prime}$ (dotted line), and $T_{\mathrm{e}} / T_{\mathrm{i}}$ (broken line).

\section{Examples}

We may assume that the experiment is done in inert gases. The results are not restricted within the usual range of the fine particle radius which is typically $1 \mu \mathrm{m} \leq r_{\mathrm{p}} \leq$ $10 \mu \mathrm{m}$. In examples shown in Sec.4.2, however, we assume that $r_{\mathrm{p}}=1 \mu \mathrm{m}$. As for $\tau_{\mathrm{ip}}$, we leave its value un- 

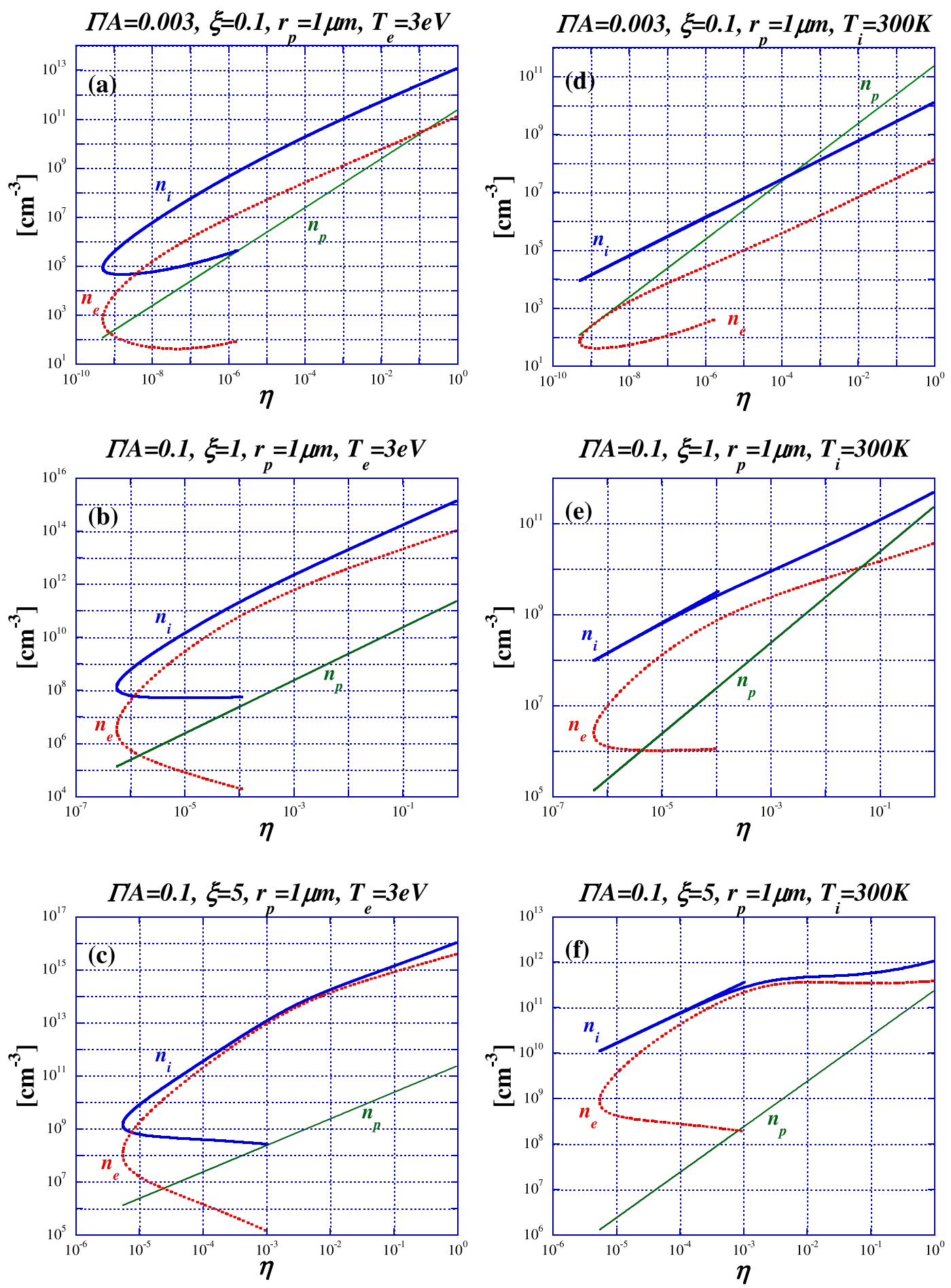

Fig. 3 Values of $n_{\mathrm{i}}\left[\mathrm{cm}^{-3}\right]$ and $n_{\mathrm{e}}\left[\mathrm{cm}^{-3}\right]$ in the case of (a-c) $T_{\mathrm{e}}=3 \mathrm{eV}$ and (d-f) $T_{\mathrm{i}}=300 \mathrm{~K}$.

specified as far as possible in order to include the cases where $T_{\mathrm{p}}$ and $T_{\mathrm{i}}$ seem to be not equal against the natural expectation $\tau_{\text {ip }}=1$.

When $r_{\mathrm{p}}$ is measured in $\mu \mathrm{m}$, we have for $n_{0}$ and $E_{0}$

$$
n_{0}=2.39 \times 10^{11} \frac{1}{\left(r_{\mathrm{p}}[\mu \mathrm{m}]\right)^{3}} \mathrm{~cm}^{-3}
$$

and

$$
E_{0}=1.44 \times 10^{-3} \frac{1}{r_{\mathrm{p}}[\mu \mathrm{m}]} \mathrm{eV}
$$

\subsection{Determination of $f_{Q}$}

The value of $f_{Q}$ depends on the species of the neutral gas through the mass of an ion. We here consider the case of Ar. The values of $f_{Q}$ are shown in Figs. 1 (a)-1(d) as functions of $\eta=\left(\Gamma / \Gamma_{0}\right)^{3}$ for typical values of $\Gamma / A$ and $\xi$ : We have solutions for $f_{Q}$ when $\Gamma / A$ is less than the critical value determined by $\xi$. There generally exist two possibilities when the solution exists for (14). It is to be noted, however, that these two solutions give possible combina- 
$\Pi A=0.003, \xi=0.1, r_{p}=1 \mu m, T_{e}=3 e V$

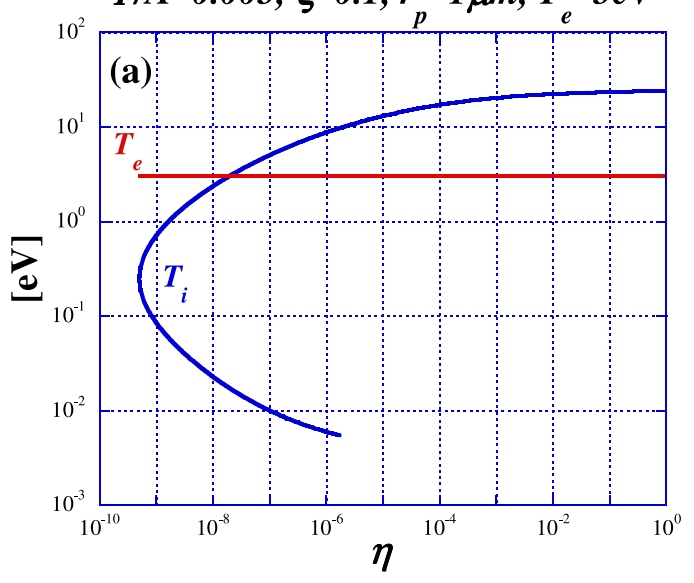

$\Pi A=0.1, \xi=1, r_{p}=1 \mu m, T_{e}=3 e V$
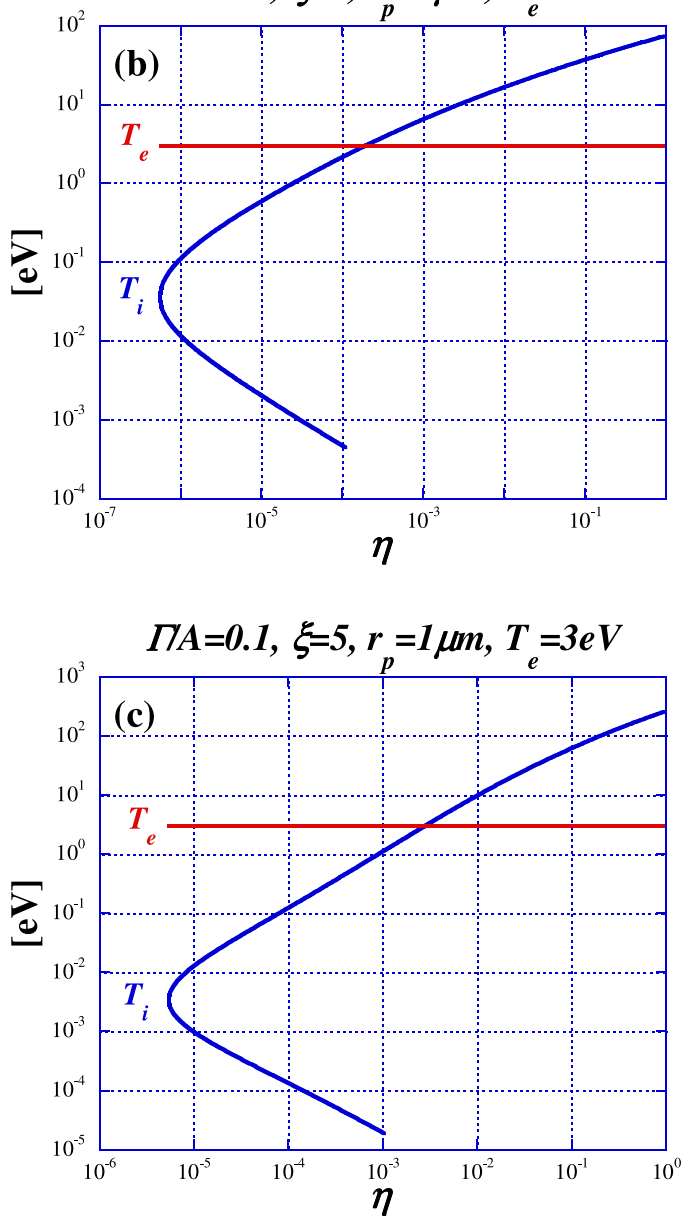

$\Pi A=0.003, \xi=0.1, r_{p}=1 \mu m, T_{i}=300 K$

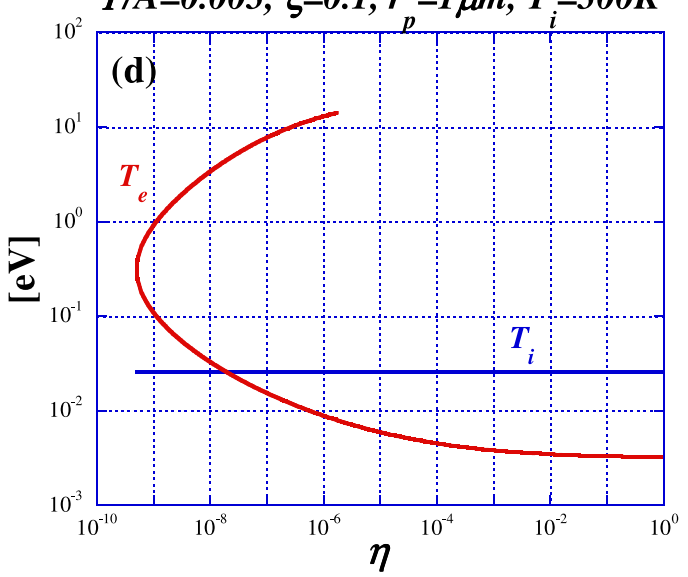

$\Pi A=0.1, \xi=1, r_{p}=1 \mu m, T_{i}=300 \mathrm{~K}$
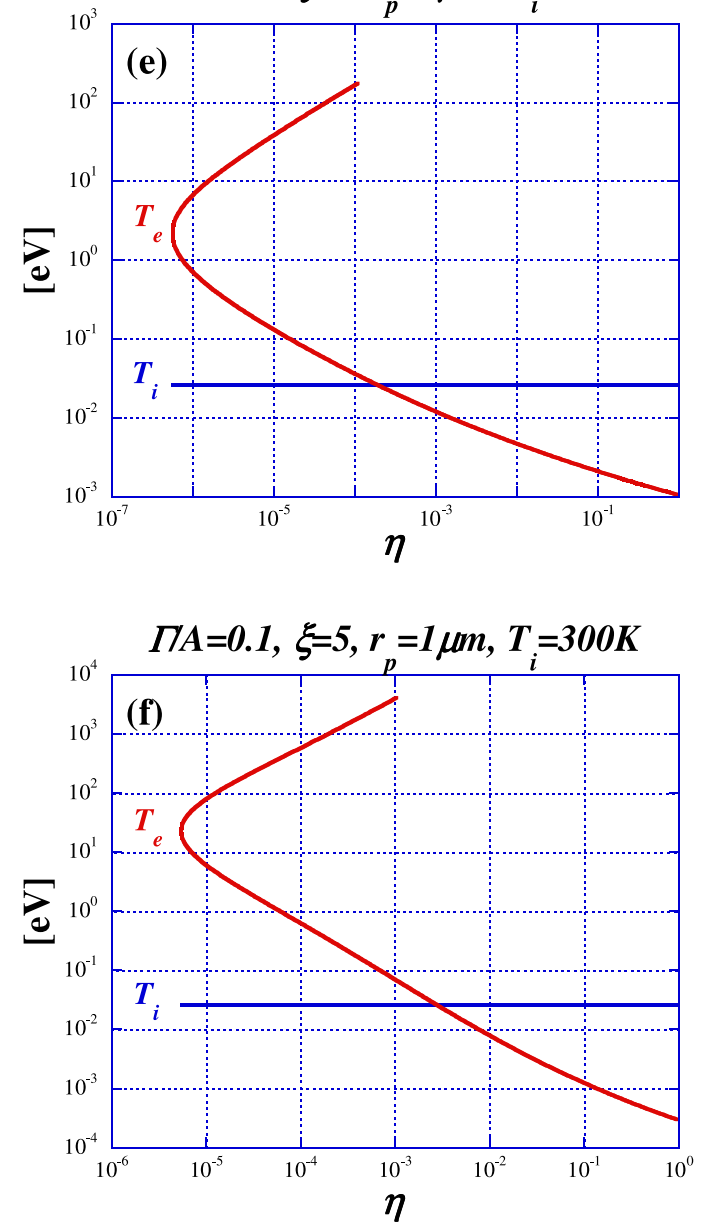

Fig. 4 Values of $T_{\mathrm{i}}[\mathrm{eV}]$ and $T_{\mathrm{e}}[\mathrm{eV}]$ in the case of (a-c) $T_{\mathrm{e}}=3 \mathrm{eV}$ and (d-f) $T_{\mathrm{i}}=300 \mathrm{~K}$.

tions of parameters and, when $n_{\mathrm{i}}, n_{\mathrm{e}}, T_{\mathrm{i}}, T_{\mathrm{e}}$, and $\tilde{r_{\mathrm{p}}}$ are specified as is in its usual application, we have only one solution for (14) if it exists.

Substituting the values of $f_{Q}$ into (22), (23), (24), and (25), values of $\left(n_{\mathrm{i}} / n_{0}\right) / A^{\prime},\left(n_{\mathrm{e}} / n_{0}\right) / A^{\prime},\left(T_{\mathrm{i}} / T_{0}\right) / A^{\prime}=$ $\tau_{\text {ip }}\left(T_{\mathrm{p}} / T_{0}\right) / A^{\prime}$, and $\left(T_{\mathrm{e}} / T_{0}\right) / A^{\prime}$ are obtained for given values of $\left(\Gamma / A, \xi, \Gamma / \Gamma_{0}\right)$. Examples of $\left(n_{\mathrm{i}} / n_{0}\right) / A^{\prime}$ and $\left(n_{\mathrm{e}} / n_{0}\right) / A^{\prime}$ are shown in Fig. 2 (a) together with $n_{\mathrm{p}} / n_{0}$ and $n_{\mathrm{e}} / Q n_{\mathrm{p}}$. In some cases, $n_{\mathrm{e}} / n_{\mathrm{i}}$ and $n_{\mathrm{e}} / Q n_{\mathrm{p}}$ are significantly smaller than unity indicating that, in these cases, the overall charge neutrality is maintained mostly by ions and negatively charged particulates.

Examples of $\left(T_{\mathrm{i}} / T_{0}\right) / A^{\prime}=\tau_{\mathrm{ip}}\left(T_{\mathrm{p}} / T_{0}\right) / A^{\prime}$ and $\left(T_{\mathrm{e}} / T_{0}\right) / A^{\prime}$ are shown in Fig. $2(\mathrm{~b})$ together with $T_{\mathrm{e}} / T_{\mathrm{i}}$. We note that the result includes the cases where $T_{\mathrm{e}} / T_{\mathrm{i}}$ is unusual. The domain of parameters realized in experiments 
$\Pi A=0.003, \xi=0.1, r_{p}=1 \mu m, T_{e}=3 e V$

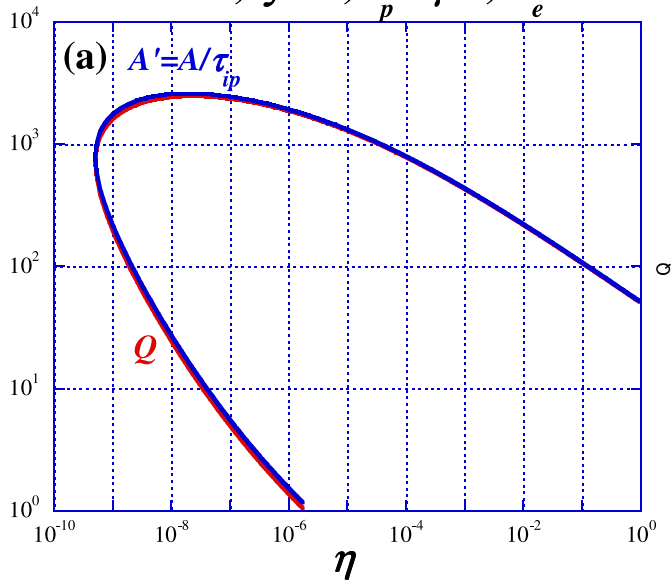

$\Pi / A=0.1, \xi=1, r_{p}=1 \mu m, T_{e}=3 e V$

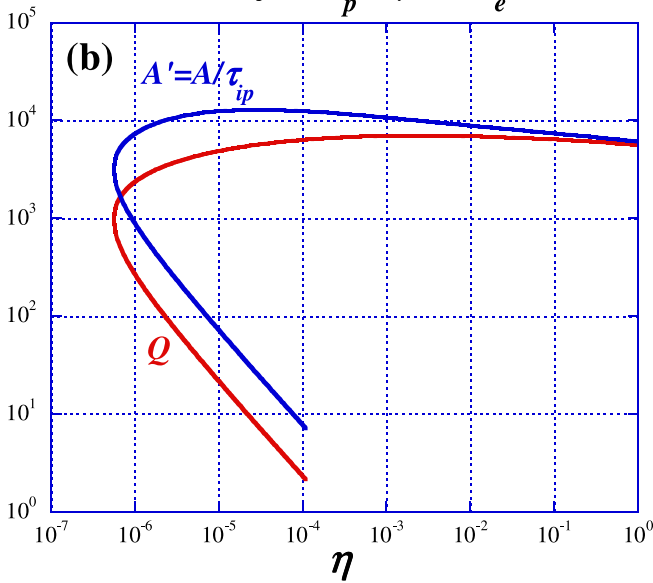

$\Pi A=0.1, \xi=5, r_{p}=1 \mu m, T_{e}=3 e V$

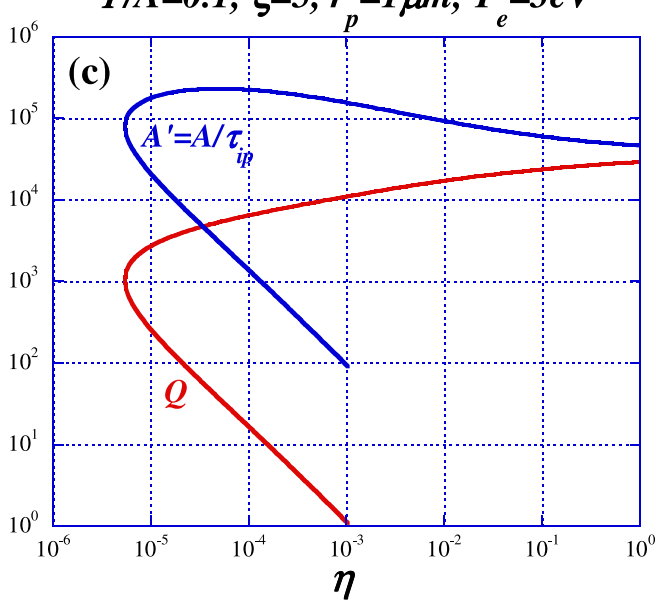

$\Pi A=0.003, \xi=0.1, r_{p}=1 \mu m, T_{i}=300 K$

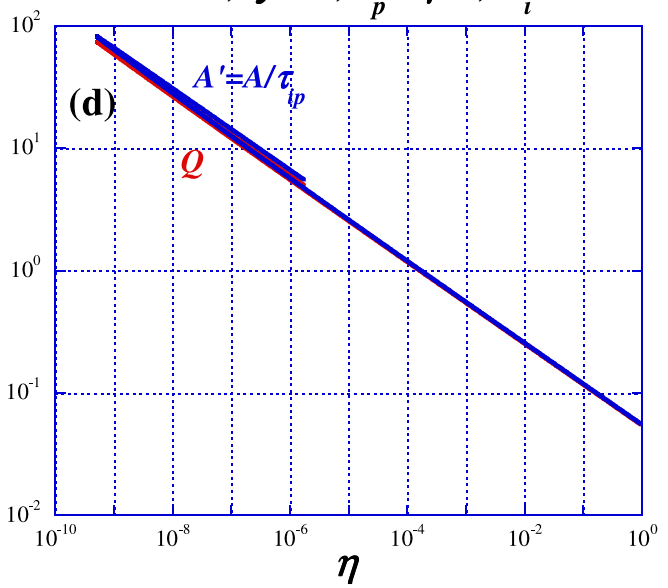

$\Pi A=0.1, \xi=1, r_{p}=1 \mu m, T_{i}=300 \mathrm{~K}$
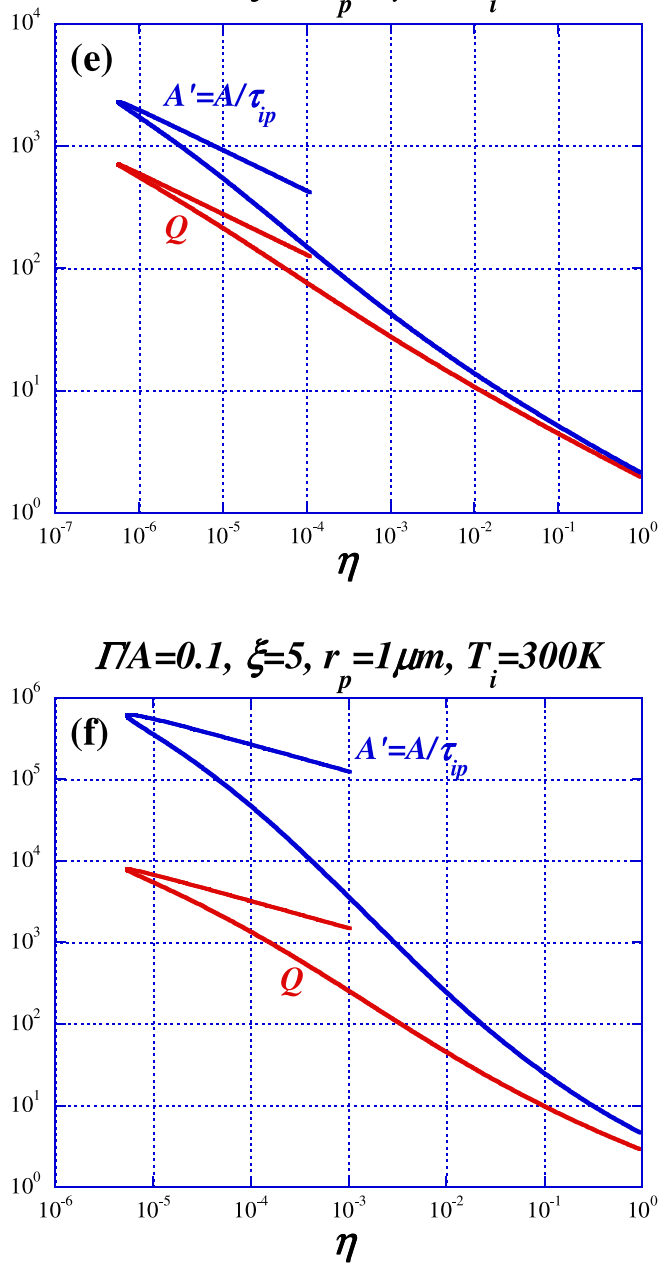

Fig. 5 Values of $A^{\prime}$ and $Q$ in the case of (a-c) $T_{\mathrm{e}}=3 \mathrm{eV}$ and (d-f) $T_{\mathrm{i}}=300 \mathrm{~K}$.

is limited when we impose some condition on this ratio corresponding to realistic situations in experiments.

In these and following examples, we include the cases with a wide range of densities and temperatures. They may be helpful to explore the possibility to extend usual limit of characteristic parameters of fine particle plasmas.

\subsection{Determination of $r_{\mathrm{p}}, A$, and $\tau_{\text {ip }}$}

Up to this point, we have not specified the particulate radius $r_{\mathrm{p}}, A$, and $\tau_{\text {ip }}$ which determine the actual (not normalized) values of densities and temperatures. For selected radius of fine particles $r_{\mathrm{p}}$, we can determine the value of $A^{\prime}=A / \tau_{\text {ip }}$ so as to adjust values of $T_{\mathrm{i}}$ or $T_{\mathrm{e}}$. According to the value of $\tau_{\text {ip }}$, all parameters are determined. 
We here show two sets of examples of values corresponding to typical values of temperatures, one with $T_{\mathrm{e}}=3 \mathrm{eV}$ and another with $T_{\mathrm{i}}=300 \mathrm{~K}$, both for the case of $r_{\mathrm{p}}=1 \mu \mathrm{m}$. Resultant values of densities, temperatures, $A^{\prime}=A / \tau_{\text {ip }}$, and $Q$ for these two sets are shown in Figs. 3 (a-c), Figs. $4(\mathrm{a}-\mathrm{c})$, and Figs. 5 (a-c) and Figs. 3 (df), Figs. 4 (d-f), and Figs. 5 (d-f), respectively.

We observe that, in the case of $(\Gamma / A=0.1, \xi=1)$, both conditions on the temperatures are satisfied when $\eta \sim$ $5.9 \times 10^{-7}$ and $\Gamma / \Gamma_{0}=\eta^{1 / 3} \sim 8.4 \times 10^{-3}$. In this case, $A \sim 2.3 \times 10^{3} \tau_{\text {ip }}, f_{Q} \sim 0.34, Q \sim 7.0 \times 10^{2}, n_{\mathrm{p}} \sim 1.4 \times$ $10^{5} \mathrm{~cm}^{-3}, n_{\mathrm{i}} \sim 1.0 \times 10^{8} \mathrm{~cm}^{-3}$, and $n_{\mathrm{e}} \sim 1.9 \times 10^{6} \mathrm{~cm}^{-3}$. Realized combination of characteristic parameters is $\xi \sim 1$, $\Gamma \sim 2.3 \times 10^{2} \tau_{\text {ip }}$, and $\Gamma_{0} \sim 2.7 \times 10^{4} \tau_{\text {ip }}$. We observe that these results include experimental parameters which are often realized in dusty plasma experiments.

The above values are for the case of $r_{\mathrm{p}}=1 \mu \mathrm{m}$. As for the $r_{\mathrm{p}}$-dependence, it should be noted that, when $\left(\Gamma / A, \xi, \Gamma / \Gamma_{0}\right)$ and the temperatures are given, $A / \tau_{\text {ip }} \propto r_{\mathrm{p}}$, $n_{\mathrm{p}} \propto r_{\mathrm{p}}^{-3}, n_{\mathrm{i}}, n_{\mathrm{e}} \propto r_{\mathrm{p}}^{-2}$, and $\Gamma / \tau_{\mathrm{ip}}, \Gamma_{0} / \tau_{\mathrm{ip}} \propto r_{\mathrm{p}}$.

\subsection{Applicability of OML theory}

It has been argued [23] that the expression of the OML theory is modified by the effect of ion-neutral collisions when the ion-neutral collision mean free path is smaller than the screening length $\lambda$. For $\lambda$, we have

$$
\frac{\lambda}{r_{\mathrm{p}}}=\frac{\Gamma_{0} / \Gamma}{\xi}=\frac{1}{\eta^{1 / 3} \xi} .
$$

On the other hand, at the pressure $p_{\mathrm{n}}$, the number density of neutral atoms (of inert gases) $n_{\mathrm{n}}$ is given by

$$
n_{\mathrm{n}}=2.41 \times 10^{14} p_{\mathrm{n}}[\mathrm{Pa}] \frac{300}{T_{\mathrm{n}}[\mathrm{K}]} \mathrm{cm}^{-3} .
$$

Here the pressure is measured in $\mathrm{Pa}$ and the neutral gas temperature $T_{\mathrm{n}}$ is measured in $\mathrm{K}$. Since the ion-neutral collision cross section $\sigma_{\text {in }}$ is $\sim 10^{-14} \mathrm{~cm}^{2}$, the ion-neutral collision mean free path is estimated as $1 / \sigma_{\text {in }} n_{\mathrm{n}} \sim 5 \times$ $10^{3}\left(p_{\mathrm{n}}[\mathrm{Pa}]\right)^{-1} \mu \mathrm{m}$ when $T_{\mathrm{n}} \sim 300 \mathrm{~K}$. The ratio $\lambda /\left(1 / \sigma_{\text {in }} n_{\mathrm{n}}\right)$ is given by

$$
\frac{\lambda}{1 / \sigma_{\mathrm{in}} n_{\mathrm{n}}} \sim \frac{r_{\mathrm{p}}[\mu \mathrm{m}] p_{\mathrm{n}}[\mathrm{Pa}]}{5 \times 10^{3} \eta^{1 / 3} \xi} \frac{300[\mathrm{~K}]}{T_{\mathrm{n}}} .
$$

We may expect the simple OML theory is applicable as a first approximation when this ratio is much smaller than unity. Rewriting the above ratio into

$$
\frac{\lambda}{1 / \sigma_{\mathrm{in}} n_{\mathrm{n}}} \sim \frac{r_{\mathrm{p}}[\mu \mathrm{m}]}{1} \frac{p_{\mathrm{n}}[\mathrm{Pa}]}{50}\left(\frac{10^{-6}}{\eta}\right)^{1 / 3} \frac{1}{\xi} \frac{300[\mathrm{~K}]}{T_{\mathrm{n}}},
$$

we see that the above condition may be satisfied in relatively high density experiments with appreciable screening $(\xi>1)$ where, for example, $\eta \xi^{3} /\left(r_{\mathrm{p}}[\mu \mathrm{m}]\right)^{3}>10^{-6}$ in the neutral gas of relatively low pressure $p_{\mathrm{n}}<50 \mathrm{~Pa}$ at the room temperature $T_{\mathrm{n}} \sim 300 \mathrm{~K}$. Our results with the simple OML theory are thus applicable in a wide domain of parameters.
In the case of low density experiments with weak screening or with high neutral gas pressures where the ratio (36) is comparable with or larger than unity, we may need to take into account the effect of ion-neutral collisions in (14). In the case of high densities, the effect of existence of other fine particles may also need consideration. In this paper, we limit ourselves within the simple OML theory leaving such cases to separate analyses.

\section{Conclusion}

Experimental parameters (the size of fine particles and densities and temperatures of fine particles, ions, and electrons) and the charge of a fine particle are explicitly expressed by characteristic parameters of fine particle plasmas modeled by the Yukawa system of finite-size particles embedded in the ambient plasma. Typical examples of the set of experimental parameters are given and the applicability of adopted charging theory is confirmed in the cases of fine particles of relatively high density in neutral gases of relatively low pressure. It is also shown for the first time that the charge neutrality condition limits the domain of fine particle plasmas such that $\xi^{2} /(\Gamma / A) \geq 3$ in terms of characteristic parameters. Though definitions of characteristic parameters are simple, the determination of experimental parameters in targeting a specific combination of characteristic parameters is not trivial. These results may be useful to design experiments in order to observe phenomena expected to occur at some specific values of the characteristic parameters [20-22].

\section{Acknowledgments}

This work has been supported in part by the Grant-inAid for Scientific Research (C) No.19540521 of the Japan Society for the Promotion of Science (JSPS).

\section{Appendix}

Let us define normalized temperatures by

$$
T_{\mathrm{i}}^{\prime}=\frac{k_{\mathrm{B}} T_{\mathrm{i}}}{e^{2} / r_{\mathrm{p}}}, \quad T_{\mathrm{p}}^{\prime}=\frac{k_{\mathrm{B}} T_{\mathrm{p}}}{e^{2} / r_{\mathrm{p}}}, \quad \text { and } \quad T_{\mathrm{e}}^{\prime}=\frac{k_{\mathrm{B}} T_{\mathrm{e}}}{e^{2} / r_{\mathrm{p}}} .
$$

Then

$$
\begin{aligned}
& Q=f_{Q} T_{\mathrm{e}}^{\prime}, \\
& \Gamma_{0}=\frac{\left(f_{Q} T_{\mathrm{e}}^{\prime}\right)^{2}}{T_{\mathrm{p}}^{\prime}},
\end{aligned}
$$

and the charge neutrality condition (10) is written as

$$
\frac{n_{\mathrm{e}}}{n_{\mathrm{p}}}=\frac{n_{\mathrm{i}}}{n_{\mathrm{p}}}-f_{Q} T_{\mathrm{e}}^{\prime}
$$

Eliminating $n_{\mathrm{e}}$ by (A4), we rewrite the definition of $\xi$, (12), into the form

$$
\frac{n_{\mathrm{i}}}{n_{\mathrm{p}}}\left(\frac{1}{T_{\mathrm{i}}^{\prime}}+\frac{1}{T_{\mathrm{e}}^{\prime}}\right)=\frac{1}{3} \xi^{2} \frac{\Gamma_{0}}{\Gamma}+f_{Q} .
$$


Also eliminating $n_{\mathrm{e}}$ from (17) and using (A3), we have

$$
A+\frac{\Gamma_{0}}{f_{Q}}=\frac{n_{\mathrm{i}}}{n_{\mathrm{p}}} \frac{T_{\mathrm{e}}^{\prime}}{T_{\mathrm{p}}^{\prime}}\left(1+\frac{T_{\mathrm{i}}^{\prime}}{T_{\mathrm{e}}^{\prime}}\right) .
$$

From (A5) and (A6), we have

$$
T_{\mathrm{e}}^{\prime}=\frac{1}{\tau_{\text {ip }}} \frac{A-1+\Gamma_{0} / f_{Q}}{(1 / 3) \xi^{2}\left(\Gamma_{0} / \Gamma\right)+f_{Q}} .
$$

Values of $T_{\mathrm{p}}^{\prime}$ and $T_{\mathrm{i}}^{\prime}$ are then given respectively by

$$
T_{\mathrm{p}}^{\prime}=\frac{\left(f_{Q} T_{\mathrm{e}}^{\prime}\right)^{2}}{\Gamma_{0}}=\frac{f_{Q}^{2}}{\Gamma_{0}} \frac{1}{\tau_{\mathrm{ip}}^{2}}\left[\frac{A-1+\Gamma_{0} / f_{Q}}{(1 / 3) \xi^{2}\left(\Gamma_{0} / \Gamma\right)+f_{Q}}\right]^{2}
$$

and

$$
T_{\mathrm{i}}^{\prime}=\tau_{\mathrm{ip}} T_{\mathrm{p}}^{\prime}=\frac{f_{Q}^{2}}{\Gamma_{0}} \frac{1}{\tau_{\mathrm{ip}}}\left[\frac{A-1+\Gamma_{0} / f_{Q}}{(1 / 3) \xi^{2}\left(\Gamma_{0} / \Gamma\right)+f_{Q}}\right]^{2} .
$$

Since we usually have $A \gg 1$, we rewrite $A-1$ into $A$.

From these results for temperatures, (A4), and (A5), densities are given as (22) and (23).

[1] For example, M.O. Robbins, K. Kremer and G.S. Grest, J. Chem. Phys. 88, 3286 (1988) and References therein.

[2] S. Hamaguchi and R.T. Farouki, J. Chem. Phys. 101, 9876 (1994).

[3] Y. Rosenfeld, Phys. Rev. E49, 4425 (1994).

[4] H. Totsuji, C. Totsuji, T. Ogawa and K. Tsuruta, Phys. Rev. E 71, 045401(R)(2005); H. Totsuji, T. Ogawa, C. Totsuji and K. Tsuruta, Phys. Rev. E 72, 036406 (2005).

[5] M. Nambu, S.V. Vladimirov and P.K. Shukla, Phys. Lett. A 230, 40 (1995).

[6] F. Melandso and J. Goree, Phys. Rev. E 52, 5312 (1995).
[7] G. Lapenta, Phys. Rev. E 66, 026409 (2002).

[8] U. Konopka, L. Ratke and H.M. Thomas, Phys. Rev. Lett. 79, 1269 (1997).

[9] U. Konopka, G.E. Morfill and L. Ratke, Phys. Rev. Lett. 84, 891 (2000).

[10] R. Kompaneets, U. Konopka, A. Ivlev, V. Tsytovich and G. Morfill, Phys. Plasmas 14, 052108 (2007).

[11] For example, S. Zhadanov, S. Nunomura, D. Samsonov and G. Morfill, Phys. Rev. E 68, 035401(R)(2003).

[12] For example, V.E. Fortov, A.V. Ivlev, S.A. Khrapak, A.G. Khrapak and G.E. Morfill, Phys. Reports 421, 1 (2005).

[13] O. Arp, D. Block, A. Piel and A. Melzer, Phys. Rev. Lett. 93, 165004 (2004).

[14] For example, V.N. Tsytovich, Y.K. Khodatev and R. Bingham, Commun. Plasma Phys. Control. Fusion, 17, 249 (1996).

[15] J.E. Daugherty, R.K. Porteous, M.D. Kilgore and D.B. Graves, J. Appl. Phys. 72, 3934 (1992).

[16] B. Deryagin and L. Landau, Acta Phys.-chim. USSR 14, 633 (1941).

[17] E.J.W. Verwey and J. Th. G. Overbeek, Theory of the Stability of Lyophobic Colloids (Elsevier, New York, 1948).

[18] For example, J.E. Allen, Phys. Scripta 45, 497 (1992); J. Goree, Plasma Sources Sci. Tech. 3, 400 (1994).

[19] O. Havnes C.K. Goertz, G.E. Morfill, E. Grun and W. Ip, J. Geophys. Res. 92, 2281 (1987).

[20] H. Totsuji, J. Phys. A: Math. Gen. 39, 4565 (2006).

[21] H. Totsuji, Non-Neutral Plasma Physics VI, Workshop on Non-Neutral Plasmas 2006, eds. M. Drewsen, U. Uggerhøj and H. Knudsen, AIP Conference Proceedings 862, (American Institute of Physics, New York, 2006) p.248.

[22] H. Totsuji, Phys. Plasmas 15, 072111 (2008).

[23] M. Lampe, R. Goswami, Z. Sternovsky, S. Robertson, V. Gavrishchaka, G. Ganguli, G. Joyce, Phys. Plasmas 10, 1500(2003); V.E. Fortov, A.V. Ivlev, S.A. Khrapak, A.G. Khrapak, G.E. Morfill, Physics Report 421, 1 (2005) Section 3 and References therein. 